\title{
Sanktionen wegen Diskriminierung ${ }^{1}$
}

\section{Europarechtliche Vorgaben}

Die Europäische Union hat allein seit dem Jahr 2000 vier Richtlinien in Kraft gesetzt, die sich mit dem Schutz vor Diskriminierung beschäftigen. Es handelt sich um die Richtlinien zur Anwendung des Gleichbehandlungsgrundsatzes ohne Unterschied der Rasse oder der ethnischen Herkunft ${ }^{2}$, zur Festlegung eines allgemeinen Rahmens für die Verwirklichung der Gleichbehandlung in Beschäftigung und Beruf ${ }^{3}$, zur Änderung der Richtlinie 76/207/EWG des Rates zur Verwirklichung des Grundsatzes der Gleichbehandlung von Männern und Frauen hinsichtlich des Zugangs zur Beschäftigung, zur Berufsbildung und zum beruflichen Aufstieg sowie in Bezug auf die Arbeitsbedingungen $^{4}$ und beim Zugang zu und der Versorgung mit Gütern und Dienstleistungen ${ }^{4 a}$. Was das Zivilrecht anbelangt, so untersagt die zuerst angeführte Richtlinie im Kern die Diskriminierung wegen »Rasse « und ethnischer Herkunft bei Abschluss und Durchführung von Schuldverträgen verschiedenster Art, die vierte diejenige wegen des Geschlechts. Die beiden anderen, im Wesentlichen allein das Arbeitsrecht betreffenden Richtlinien umfassen darüber hinaus die Diskriminierung wegen der Religion oder Weltanschauung, einer Behinderung, des Alters, der sexuellen Ausrichtung sowie des Geschlechts.

Zur Umsetzung der das allgemeine Vertragsrecht betreffenden Richtlinie hatte das Bundesministerium der Justiz bereits im Jahr 2001 einen Diskussionsentwurf ${ }^{5}$ erarbeitet, der über die Diskriminierung wegen »Rasse« und ethnischer Herkunft hinaus eine Reihe weiterer, in jener Richtlinie nicht vorgesehener Diskriminierungsverbote für das Privatrecht vorsah. Dieser Entwurf wurde nach teils heftiger Kritik ${ }^{6}$ schließlich zurückgezogen. Für die damit noch ausstehende Umsetzung, zu der am 21.1.2005 ein neuer Entwurf ${ }^{6 a}$ im Bundestag eingebracht wurde, enthalten die Richtlinien eine Reihe klarer und bindender Vorgaben. So wird etwa die Einrichtung von Anti-Diskriminie-

1 Der Beitrag beruht auf Überlegungen, die der Verfasser auf dem Symposium »Reform des Diskriminierungsschutzes in Europa - Wo steht Deutschland?« am 24.6.2004 in der Vertretung der Europäischen Kommission in Berlin vorgetragen hat.

2 Richtlinie 2000/43/EG des Rates vom 29.6.2000, ABl. EG Nr. L 180, S. 22; dazu Nickel, NJW 2001, 2668 ff.; Schiek, AuR 2003, 44 ff.

3 Richtlinie 2000/78/EG des Rates vom 29.11.2000, ABl. EG Nr. L 303, S. 16; dazu eingehend J. Mohr, Schutz vor Diskriminierungen im Europäischen Arbeitsrecht, 2002; zu beiden Richtlinien s. J.-H. Bauer, NJW 2001, 2672 ff.; Leuchten, NZA 2002, 1254 ff.; Neuner, JZ 2003, 57 ff.; Thüsing, NZA 2001, $1061 \mathrm{ff.}$

4 Richtlinie 2002/73/EG des Europäischen Parlaments und des Rates vom 22.9.2002, ABl. EG Nr. L 269, S. 15; dazu Rust, NZA 2003, 72 ff.

4a Richtlinien 2004/113/EG des Rates vom 13.12.2004, ABl. EG Nr. L 373, S. 37.

5 Abgedruckt in DB 2002, $470 \mathrm{f}$.

6 S. nur Säcker, ZRP 2002, 286 ff.; von Koppenfels, WM 2002, 1489 ff.

6a BT-Drucks. 15/4538, abgedruckt in NJW Heft 1-2/2005, S. XXXV. 
rungsstellen vorgeschrieben, zudem müssen bestimmte Verhaltensanforderungen für Arbeitgeber vorgesehen werden. ${ }^{7}$

Was hingegen die Sanktionen einer unzulässigen Diskriminierung angeht, so bleibt es den Mitgliedstaaten überlassen, diese selbst festzulegen. Allerdings sind die nationalen Gesetzgeber in der Wahl der Instrumente nicht völlig frei. Vielmehr ist es europarechtlich geboten, effektive Sanktionen vorzusehen. In den Richtlinien heißt es hierzu, dass die Sanktionen »wirksam, verhältnismäßig und abschreckend ${ }^{8}$ sein müssen. Dieses Gebot hat den deutschen Gesetzgeber schon einmal dazu veranlasst, eine ursprünglich vorgesehene Sanktion zu modifizieren, nämlich beim geschlechtsbezogenen Benachteilungsverbot im Arbeitsrecht (§ 611 a BGB). Die hier zunächst eingeführte Sanktion, dass ein bei der Einstellung benachteiligter Arbeitnehmer lediglich das negative Interesse als Schadensersatz verlangen konnte, also regelmäßig nur die Bewerbungskosten (»Portoparagraph $\aleph^{9}$ ), hielt der EuGH ${ }^{10}$ für zu schwach. Seit 1994 sieht § 611 a II BGB statt dessen einen Anspruch auf »angemessene Entschädigung in Geld « vor. Klargestellt hat der EuGH ${ }^{11}$ zudem, dass ein derartiger Entschädigungsanspruch nicht an ein Verschuldenserfordernis geknüpft werden darf. Diskriminierendes Verhalten bildet damit neben der Gefährdungshaftung eine weitere Fallgruppe einer verschuldensunabhängigen gesetzlichen Haftung. ${ }^{12}$

Schließlich folgt aus europarechtlichen Grundsätzen auch, dass der deutsche Gesetzgeber sich nicht auf die Position zurückziehen darf, das geltende Privatrecht enthalte bereits jetzt in Gestalt allgemeiner Regeln wie insbesondere der $\$ \S 823,826$ BGB und des quasi-negatorischen Unterlassungsanspruchs analog $\S 1004$ BGB $^{13}$ sowie $\S 138 \mathrm{BGB}^{14}$ wirksame Sanktionen unzulässiger Diskriminierung. ${ }^{15}$ Eine schlichte Notifizierung dieser Regeln würde den Anforderungen an eine ordnungsgemäße Umsetzung der Richtlinien schon deshalb nicht genügen, weil dem Rechtsadressaten aus jenen allgemeinen Vorschriften seine europarechtlich gewährleisteten Rechte nicht hinreichend klar erkennbar werden. ${ }^{16}$ Entscheidend kommt hinzu, dass die Voraussetzungen und Rechtsfolgen eines auf die allgemeinen deliktsrechtlichen Regeln gestützten Anspruchs im Bereich der Diskriminierung durchaus umstritten sind. So kann es keineswegs als anerkannt gelten, dass bei diskriminierender Verweigerung eines Vertragsschlusses ein Kontrahierungszwang besteht. ${ }^{17}$ Sofern sich hinge-

$7 \mathrm{Zu}$ den Richtlinienvorgaben im Einzelnen s. die in Fn. 3 und 4 Genannten.

8 Art. 17 S. 2 der Richtlinie 2000/78/EG; Art. 15 S. 2 der Richtlinie 2000/43/EG; Art. 8 d S. 2 der Richtlinie 76/207/EWG i.d.F. der Richtlinie 2002/73/EG, im selben Sinne Art. 8 II der Richtlinie 2004/113/EG.

9 Freis, NJW 1998, 2779; Zuleeg, RdA 1984, 325 (327).

10 EuGH NJW 1984, 2021.

11 EuGH NJW 1997, 1839 (1840, sub 17-22) [Nils Draehmpaehl/Urania Immobilienservice $O H G]$.

12 Zutr. Buchner, in: MünchHdbArbR, 2. Aufl. 2000, § 40 Rdnr. 201 (zu § 611 a BGB).

13 S. dazu noch unten bei Fn. 29.

14 Dazu T. Bezzenberger, AcP 196 (1996), 395 (421 ff.).

15 In dieser Richtung aber Picker, JZ 2003, 540 (545); mit Einschränkungen auch von Koppenfels, WM 2002, 1489 (1494 ff.); vgl. ferner Manger, GE 2004, 328.

16 S. zu den Anforderungen EuGH NJW 2001, 2244. 
gen von der Rechtsprechung aufgestellte Regeln heranziehen lassen, erfüllen diese nicht ohne weiteres die europarechtlichen Anforderungen an die Effektivität und Abschreckungswirkung der Sanktion. Insoweit sei hier nur daran erinnert, dass ein Anspruch auf Ersatz des immateriellen Schadens wegen Verletzung des allgemeinen Persönlichkeitsrechts nach der Rechtsprechung des BGH eine besonders schwerwiegende Beeinträchtigung voraussetzt; ${ }^{18}$ die Richtlinien sehen eine derartige Einschränkung hingegen nicht vor. Schließlich sind auch das deliktsrechtliche Verschuldenserfordernis für die Zuerkennung eines Zahlungsanspruchs und die Anwendung der allgemeinen Beweislastregeln nicht richtlinienkonform.

\section{Mögliche Sanktionen}

\section{Sanktionen bei diskriminierungsbedingter Verweigerung eines Vertragsschlusses}

Was die dem Gesetzgeber zur Wahl stehenden Sanktionen angeht, so soll hier zunächst der besonders gravierende Fall betrachtet werden, dass bereits der Abschluss eines Vertrages aus unter das Diskriminierungsverbot fallenden Gründen verweigert wird. Eine auf den ersten Blick nahe liegende Sanktion besteht hier in einem Kontrahierungszwang. Dabei geht es nicht um eine aus dem öffentlichen Angebot herrührende rechtsgeschäftliche Pflicht; ${ }^{19}$ vielmehr steht eine normativ begründete Verpflichtung in Rede. Nach geltendem Recht ist ein solcher Kontrahierungszwang bei geschlechtsbezogener Diskriminierung im Arbeitsrecht ausdrücklich ausgeschlossen (§ 611 a II a.E. BGB). Auch schreibt der Richtliniengeber ihn - wie gesagt - nicht bindend vor. ${ }^{20}$ Das lässt zumindest aufhorchen. Statt des Kontrahierungszwangs kommt auch ein Beseitigungsanspruch in Betracht, wie er schon im Regierungsentwurf von $2001^{21}$ unter dem der zivilrechtlichen Terminologie fremden ${ }^{22}$ und inhaltlich eher zum Schadensersatz passenden Begriff »Folgenbeseitigungsanspruch« vorgesehen war. Der Betroffene kann

17 Gewichtige dogmatische Bedenken etwa bei Neuner, JZ 2003, 57, 61; abl. im Grundsatz auch F. Bydlinski, AcP 180 (1980), 1 (14 f., 44 f.); von Koppenfels, WM 2002, 1489 (1495); einschränkend Busche, Privatautonomie und Kontrahierungszwang, 1999, S. 214 ff., 295; einen generellen Kontrahierungszwang bei ethnischer Diskriminierung bejahend hingegen etwa T. Bezzenberger, AcP 196 (1996), 395 (429 f.). Zum Streitstand s. Kramer, in: MünchKomm-BGB, 4. Aufl. 2001, Vor § 145 Rndr. 13 ff.; Staudinger/Bork, BGB, Neubearb. 2003, Vor $\S \S 145-156$ Rdnr. 24 (Rechtslage bei »rassischer« Diskriminierung sei »unklar«).

18 BGHZ 35, 363 (367 ff.); Staudinger/Richardi/Annuß, BGB, 13. Bearb. 1999, § 611 a Rdnr. 68. S. auch (zur Nichtaufnahme des allgemeinen Persönlichkeitsrechts in § 253 II BGB n.F.) BT-Drs. 14/7752, S. 25.

19 In dieser Richtung Baer, ZRP 2002, 290 (293), die von einer Bindung an das Angebot spricht; zutr. dagegen bereits F. Bydlinski, AcP 180 (1980), 1 (40).

20 S. auch Thüsing, NJW 2003, 3441 (3444, »keine Mindestsanktion«). Daraus folgert Nickel, NJW 2001, 2668 (2671) offenbar, dass er auch nicht einzuführen ist; indessen überlassen die Richtlinien diese Entscheidung den nationalen Gesetzgebern.

$21 \S 319$ e I BGB-E (abgedr. in DB 2002, 471).

22 Krit. auch von Koppenfels, WM 2002, 1489 (1495); Neuner, JZ 2003, 57 (66); Wiedemann/ Thüsing, DB 2002, 463 (469); a.A. Kilian, AcP 180 (1980), 47 (82). 
demnach verlangen, dass die Beeinträchtigung beseitigt wird. Dies läuft dann, wenn die Diskriminierung in der Verweigerung eines Vertragsschlusses besteht und ein solcher immer noch möglich ist, gleichfalls auf einen Kontrahierungszwang hinaus. ${ }^{23}$ Ferner ist an einen Schadensersatzanspruch zu denken. Auf Grundlage des geltenden Rechts wird vielfach dahin argumentiert, dass auch er zu einem Kontrahierungszwang führen könne. ${ }^{24}$ Nach dem Grundsatz der Naturalrestitution (§ 249 I BGB) sei der Geschädigte so zu stellen ist, wie er stünde, wenn das diskriminierende Verhalten unterblieben wäre. Liegt dieses Verhalten gerade in der Verweigerung eines Vertragsschlusses, bestehe daher auch der Schadensersatz in einer Pflicht zum Vertragsschluss. Diese Erwägung ist mit Recht aus schadensersatzrechtlichen Gründen in Zweifel gezogen worden. Beim Kontrahierungszwang geht es nämlich nicht um die Kompensation eines bereits eingetretenen Schadens, sondern um die primäre Durchsetzung einer Verhaltenspflicht. ${ }^{25}$ Stattdessen kommt ein Anspruch auf »angemessene Entschädigung in Geld « in Betracht, wie er für den Fall der geschlechtsbezogenen Benachteiligung bereits in $§ 611$ a II BGB vorgesehen ist. Für diese Regelung ist es umstritten, ob der Anspruch neben immateriellen auch materielle Schäden, also Vermögensschäden, umfasst. ${ }^{26}$ Eine (konsolidierende) Neuregelung sollte hier in jedem Fall Klarheit schaffen.

Als weitere Sanktionsmöglichkeit kommt (zusätzlich zu den schon bestehenden strafrechtlichen Ehrschutztatbeständen und unter geringeren Voraussetzungen) eine Verfolgung als Ordnungswidrigkeit in Betracht. ${ }^{27} \mathrm{Im}$ Vergleich dazu ist es indessen das modernere, effizientere Mittel, den Betroffenen selbst zivilrechtliche Ansprüche zu geben. Dies soll hier nicht vertieft werden. Letzteres gilt auch für einen Unterlassungsanspruch. Er gewinnt seine Bedeutung nicht in der konkreten Situation eines von einer Diskriminierung bereits Betroffenen, sondern bietet lediglich einen die erforderliche effektive Sanktion gleichsam flankierenden vorbeugenden Schutz bei Wiederholungsgefahr.

\section{Sanktionen bei sonstiger Diskriminierung}

Diskriminierung kommt nicht nur beim Vertragsschluss vor. Zu denken ist darüber hinaus insbesondere an Gestaltungserklärungen wie Kündigung und Rücktritt. Auch ein sonstiges Verhalten im Rahmen der Vertragsabwicklung kann diskriminierend wirken, etwa die Verweigerung üblicher Vorzüge wie Kulanzleistungen. Ein Kontrahierungszwang ist in solchen Fällen naturgemäß von vornherein keine geeignete Sanktion. In Betracht kommen aber andersartige Ansprüche auf Beseitigung, Schadenser-

23 Zutr. von Koppenfels, WM 2002, 1489 (1490 f., zum Gesetzentwurf von 2001).

24 S. nur Thüsing, NJW 2003, 3441 (3445); Wiedemann/Thüsing, DB 2002, 463 (469).

25 Eingehend Busche (Fn. 17), S. 220 ff.; s. auch T. Bezzenberger, AcP 196 (1996), 395 (427 f.); F. Bydlinski, AcP 180 (1980), 1 (13); Kilian, AcP 180 (1980), 47 (82); Neuner, JZ 2003, 57 (61).

26 Dafür die h.M.; s. etwa Annuß, NZA 1999, 738 (741); Palandt/Putzo, BGB, 64. Aufl. 2005, $\S 611$ a Rdnr. 19; Müller-Glöge, in: MünchKomm-BGB, 4. Aufl. 2005, § 611 a Rdnr. 61; Schlachter, in: ErfKoArbR, 4. Aufl. 2004, § 611 a BGB Rdnr. 37; dagegen [zur Vorgängervorschrift] Oetker, ZIP 1997, 802 (803); Steinmeister, PersR 1995, 9 (10).

27 Dafür etwa Аnпи $\beta$, NZA 1999, 738 (744, zu § 611 a I BGB). 
satz oder Entschädigung in Geld. Zudem ist für Gestaltungserklärungen deren Nichtigkeit nach $§ 134$ BGB i.V. mit dem zu kodifizierenden Diskriminierungsverbot zu erwägen.

\section{Stellungnahme}

Der Überblick hat gezeigt, dass eine ganze Reihe von Sanktionen in Betracht kommen. Die folgende Stellungnahme konzentriert sich auf die wesentlichen Grundentscheidungen, die der deutsche Gesetzgeber zu treffen hat.

\section{Kontrahierungszwang oder Entschädigung in Geld?}

Kontrahierungszwänge kennt das geltende Recht bereits. Es würde sich also nicht um einen völligen Fremdkörper im deutschen Recht handeln. Allerdings sind die anerkannten Fallgruppen bislang eng umgrenzt. Es geht um Fälle, in denen ein Marktteilnehmer auf eine Leistung angewiesen ist und sich einem überragend marktmächtigen (marktbeherrschenden) Anbieter gegenüber sieht. ${ }^{28}$ Weiter gehend wird bereits auf der Grundlage des geltenden Rechts ein allgemeiner Kontrahierungszwang auch bei Diskriminierungen vorgeschlagen. Gestützt wird er auf eine Analogie zu den gesetzlichen Spezialregeln, auf $\S 826$ BGB oder auf einen quasi-negatorischen Unterlassungsanspruch. ${ }^{29}$ Jener Ansatz erscheint indessen nur so lange als unverzichtbar, wie keine effektive Alternative als Sanktion in Betracht kommt und Diskriminierungen somit anderenfalls mehr oder minder sanktionslos blieben. ${ }^{30}$ Eine solche Alternative steht nun aber zur Wahl, nämlich mit dem Anspruch auf angemessene Entschädigung in Geld, also auf Ersatz zumindest auch eines immateriellen Schadens. Damit stellt sich die Frage, welche dieser beiden Sanktionen vorzugswürdig ist.

Der Gesetzgeber hat diese Frage - wie bereits erwähnt - schon einmal beantwortet und eine Entschädigung in Geld dem Kontrahierungszwang vorgezogen, nämlich beim geschlechtsbezogenen Benachteiligungsverbot im Arbeitsrecht ( $\$ 611$ a II BGB). Damit ist freilich noch keine bindende Vorgabe im Sinne einer Grundsatzentscheidung geschaffen. Der gesetzgeberische Verzicht darauf, den Arbeitgeber zum Abschluss des Arbeitsvertrages zu zwingen, mag nämlich jedenfalls auch darauf gestützt werden, dass ein Kontrahierungszwang sich hier zu lasten anderer Arbeitnehmer auswirken könnte, auf deren Einstellung der Arbeitgeber dann verzichten oder denen er kündigen würde. ${ }^{31}$

Es soll hier auch nicht abstrakt erörtert werden, ob die mit einem Kontrahierungszwang verbundene Einschränkung der Privatautonomie hinnehmbar ist oder nicht. Das Augenmerk soll vielmehr auf drei konkrete Aspekte gelenkt werden.

28 S. etwa § 10 I EnWG, § 22 PBefG, § 8 PostG, § 5 PflVersG, § 87 IV UrhG, ferner das kartellrechtliche Diskriminierungsverbot gem. § 20 GWB. Eingehend Busche (Fn. 17), S. 299 ff.

29 Zum Meinungsstand s. T. Bezzenberger, AcP 196 (1996), 395 (427 ff.); Erman/Armbrüster, BGB, 11. Aufl. 2004, Vor $\S 145$ Rdnr. 28.

30 Vgl. auch Staudinger/Richardi/Annuß (Fn. 18), § 611 a Rdnr. 68 (»Notlösung«).

31 S. nur Schlachter, in: ErfKoArbR (Fn. 26), § 611 a BGB Rdnr. 37. 
a) Schutzinteresse des Diskriminierten. Der erste Aspekt betrifft das Schutzinteresse des Diskriminierten. Hierzu gehen die Einschätzungen weit auseinander. Teils ${ }^{32}$ wird geltend gemacht, dieses Interesse sei gerade auf den Vertragsschluss gerichtet; schließlich gehe es um den Zugang zu Wirtschaftsgütern. Andere ${ }^{33}$ gehen davon aus, im Vordergrund stehe ein Ausgleich für das verletzende Verhalten, für die Herabwürdigung der Person. Bei lebensnaher Betrachtung sei das Schutzinteresse daher auf Ausgleich durch Geldzahlung gerichtet, nach Art eines Schmerzensgeldes.

Diese Positionen werden meist nicht näher begründet. Eine sachorientierte Entscheidung sollte bei der Frage ansetzen, ob wirklich der Zugang zu Wirtschaftsgütern, die Teilhabe am Markt im Mittelpunkt des Interesses steht. Die Frage zu stellen, heißt sie zu verneinen. In der Regel kann der Diskriminierte sich eine vergleichbare Leistung schnell und problemlos anderweitig beschaffen. Die in einer Marktwirtschaft typischerweise vorhandene Vielzahl von Anbietern sind nicht durch »Diskriminierungskartelle « miteinander verbunden. ${ }^{34}$ Dies spricht zugleich auch dagegen, unter dem Gesichtspunkt der Zumutbarkeit ${ }^{35}$ ausschließlich für »Massengeschäfte des täglichen Lebens « einen Kontrahierungszwang einzuführen; gerade insoweit ist kein Schutzbedürfnis ersichtlich. ${ }^{36}$ Wo die Lage hingegen wegen überragender Marktmacht eines Anbieters anders ist, kommt dem Betroffenen wie jedem anderen Nachfrager der darauf gestützte allgemeine Kontrahierungszwang zugute. Es bleiben die Fälle, in denen dem Betroffenen der Vertragsgegenstand als unersetzbar erscheint. Beispiele bieten eine ganz bestimmte Mietwohnung oder ein Unikat wie etwa ein Kunstwerk. In solchen Fällen wird der Gegner freilich regelmäßig schon anderweitig über den Vertragsgegenstand verfügt haben, bevor der Diskriminierte gerichtlich vorgehen kann. In diesem Fall kommt ohnehin nur noch Schadensersatz in Betracht.

Das wahre Problem bei der Diskriminierung liegt nicht in einer Verhinderung des Marktzugangs, sondern in der persönlichen Herabwürdigung des Diskriminierten. ${ }^{37}$ Nicht von ungefähr wird Diskriminierung immer wieder mit einer Verletzung des allgemeinen Persönlichkeitsrechts in Verbindung gebracht. ${ }^{38}$ Der darin liegende Angriff auf die Person soll ausgeglichen werden. Dafür ist - neben rechtlich nicht erzwingbarem Verhalten wie einer Entschuldigung - ein finanzieller Ausgleich sachgerecht. Dies allein bedeutet freilich noch nicht, dass ein Kontrahierungszwang abzulehnen ist; schließlich könnte man auch ein Wahlrecht oder eine Kombination beider Sanktionen $^{39}$ einführen. Es kommen indessen zwei weitere Aspekte hinzu.

32 S. Braun, AnwBl 2002, 569 (571: das Interesse richte sich in erster Linie darauf, diskriminierungsfrei behandelt zu werden); tendenziell auch T. Bezzenberger, AcP 196 (1996), 395 (427).

33 Implizit Nickel, NJW 2001, 2668 (2671).

34 Insoweit zutr. Adomeit, NJW 2002, 1622 (1623).

35 S. dazu Baer, ZRP 2002, 290 (293); vgl. auch dies., ZRP 2001, 500 (503).

36 Vgl. bereits F. Bydlinski, AcP 180 (1980), 1 (14 f., 44 f.).

37 In dieser Richtung auch Busche (Fn. 17), S. 287 f. (im Kontext von $§ 611$ a BGB).

38 Vgl. BAG AP § 611 a BGB Nr. 5 (Anstellung im Tierheim); Müller-Glöge, in: MünchKomm-BGB (Fn. 26), § 611 a Rdnr. 59 (ein darauf gestützter deliktsrechtlicher Anspruch sei »theoretisch begründbar«). 
b) Effektivität der Sanktion. Nimmt man einen Kontrahierungszwang an, so trifft dies den Vertragsgegner jedenfalls wirtschaftlich weniger hart als eine Zahlungspflicht. Schließlich geht es um Verträge, die der Gegner ohne die Diskriminierung abgeschlossen hätte, aus denen er sich also typischerweise einen Gewinn erhofft. Dieser Gewinn bliebe ihm bei einem Kontrahierungszwang erhalten, während er anderenfalls einem nicht diskriminierenden Marktteilnehmer zugute kommt, mit dem der Benachteiligte ein Deckungsgeschäft tätigt. ${ }^{40}$ Umgekehrt bietet eine angemessene Entschädigung in Geld die Möglichkeit, über die Höhe des Betrages erforderlichenfalls besonders nachhaltig auf den diskriminierenden Marktteilnehmer einzuwirken.

c) Befriedungsfunktion des Privatrechts. Der letzte und bedeutsamste Aspekt richtet den Blick auf die Folgewirkungen des Kontrahierungszwangs für das Verhältnis der Parteien. Eine der wesentlichen Aufgaben der Privatrechtsordnung ist neben der Entlastungsfunktion und der Leitbildfunktion für einen gerechten Interessenausgleich ihre Befriedungsfunktion (Konfliktlösungsfunktion ${ }^{41}$ ). Ob diese Funktion optimal erreicht wird, wenn einem Beteiligten ein Vertragsschluss aufgezwungen wird, ist höchst zweifelhaft. Im Stadium der Erfüllung eines Vertrages eröffnen sich nämlich eine Vielzahl von möglichen Streitquellen. Ein nicht aus privatautonomer Entscheidung, sondern allein infolge eines Kontrahierungszwangs geschlossener Vertrag steht von vornherein unter keinem guten Stern. Es muss damit gerechnet werden, dass beide Seiten kaum eine Gelegenheit auslassen werden, die möglichen Streitquellen aufzugreifen und Folgestreitigkeiten auszulösen, etwa über Leistungsstörungen. Der Gegner wird häufig nichts unversucht lassen, um mithilfe von Instrumenten wie Rücktritt oder Kündigung die ihm aufgezwungene Vertragsbindung zu beseitigen.

Zwar besteht eine Gefahr erhöhter Folgestreitigkeiten im Grunde bei jedem Kontrahierungszwang, also auch in den bereits anerkannten Fällen überragender Marktmacht $^{42}$. Allerdings gibt es zwei Unterschiede ${ }^{43}$ : Die Streitgefahr ist in jenen Fällen tendenziell deutlich geringer, da das Verhältnis der Vertragsparteien meist unpersönlich und damit weniger spannungsgeladen ist (Großunternehmen; andersartige Ablehnungsgründe). Vor allem aber ist der Diskriminierte - wie aufgezeigt - regelmäßig objektiv nicht gerade auf den betreffenden Anbieter angewiesen. Angesichts dieser Unterschiede ist es auch nicht aus Gründen der Systematik geboten, das Regelungsmodell des $\S 20 \mathrm{GWB}^{44}$ auf die Diskriminierungstatbestände zu übertragen, zumal da

39 So der neue Entwurf (Fn. 6a) in § 22 ADG und bereits der Gesetzentwurf von Abgeordneten und der Fraktion der SPD zur Anpassung des geschlechtsbedingten arbeitsrechtlichen Benachteiligungsverbots an das EU-Recht, BT-Drs. 13-7896 und dazu Freis, NJW 1998, 2779 (2782). Vgl. auch Neuner, JZ 2003, 57 (64).

40 Dies übergeht Baer, ZRP 2002, 290 (293).

41 Th. Raiser, Das lebende Recht. Rechtssoziologie in Deutschland, 3. Aufl. 1999, S. 198 f.; Rehbinder, Rechtssoziologie, 5. Aufl. 2003, Rdnr. 96, 110; vgl. auch Kißler, Recht und Gesellschaft, 1984, S. 99.

42 S. dazu die Nachw. in Fn. 28.

43 Die Unterschiedlichkeit betonen auch von Koppenfels, WM 2002, 1489 (1492); T. Bezzenberger, AcP 196 (1996), 395 (430 f.).

44 Zum daraus folgenden Kontrahierungszwang s. Staudinger/Bork (Fn. 17), Vor §§ 145-156 Rdnr. 19. 
das gleichfalls bereits vorhandene Modell des $§ 611$ a II BGB, das einen Diskriminierungstatbestand betrifft, in die entgegengesetzte Richtung weist.

Als Fazit bleibt festzuhalten, dass ein gesetzlicher Kontrahierungszwang in den Diskriminierungsfällen die Befriedungsfunktion des Privatrechts gefährden würde, ohne dass ein Schutzinteresse des Betroffenen dies gebietet. Ein Kontrahierungszwang - der stets einen besonders weitreichenden Eingriff in die Privatautonomie darstellt ${ }^{45}$ sollte daher weder ausdrücklich noch in Gestalt eines Beseitigungsanspruchs eingeführt werden. Beseitigungsansprüche haben, nebenbei bemerkt, ohnehin auch in der praktischen Rechtsanwendung ihre Tücken: So ist es bis heute lebhaft umstritten, wie der Beseitigungsanspruch aus $§ 1004$ BGB von deliktsrechtlichen Schadensersatzansprüchen abzugrenzen ist. ${ }^{46}$ Vorzugswürdig ist ein Anspruch auf angemessene Entschädigung in Geld. Damit ist auch ein systematischer Gleichklang zu $§ 611$ a II BGB hergestellt. $^{47}$

\section{Umfang des Ausgleichs in Geld}

\section{a) Materieller und immaterieller Schaden.}

Spricht man sich mit der hier vertretenen Ansicht für einen Ausgleich in Geld als Sanktion aus, so stellt sich die Frage nach dem Umfang des Anspruchs. Man sollte ihn auf materielle wie auch auf immaterielle Schäden erstrecken. Die Richtlinien sollen den Betroffenen nämlich umfassend vor seiner unzulässigen Ausgrenzung und den damit verbundenen Nachteilen schützen. Das muss sich in den Sanktionen niederschlagen. Hätte die andere Seite ohne die Diskriminierung einen Vertrag geschlossen, so muss sie daher auch für den infolge der Verweigerung entstandenen Vermögensschaden einstehen. ${ }^{48}$ Was die Regelungstechnik angeht, so sollte der Gesetzgeber sich nicht darauf beschränken, die Neuregelung nur auf immaterielle Schäden zu beziehen und wegen der Vermögensschäden auf die allgemeinen Vorschriften zum Schadensersatz zu verweisen. Aus diesen Vorschriften (culpa in contrahendo [\$§ 311 II, 241 II, 280 I BGB], $\S \S 823$ und 826 BGB) wird nämlich ein Schadensersatzanspruch tatbestandlich keineswegs stets folgen. Dies gilt mit Blick auf das Verschuldenserfordernis insbesondere bei unbewusster und mittelbarer Diskriminierung. Europarechtlich darf der Ersatzanspruch jedoch gerade nicht an ein Verschulden geknüpft werden. Zudem könnte über einen Schadensersatzanspruch ein Kontrahierungszwang qua Naturalrestitution hergeleitet werden, was - wie aufgezeigt - vor allem wegen der dann drohenden Folgestreitigkeiten nicht sachgerecht ist.

b) Kriterien für die Bemessung des immateriellen Schadens. Ist eine angemessene Entschädigung geschuldet, so können (wie beim Schmerzensgeld) die Umstände des

45 H. Ehlers, Die Problematik des Kontrahierungszwangs in der Wettbewerbsordnung, 1979, S. 16; Mestmäcker, JZ 1964, 441 (443): »schärfster Eingriff « in die Vertragsfreiheit.

46 S. dazu Armbrüster, NJW 2003, 3087 (3088 f.).

47 Insoweit zutr. Neuner, JZ 2003, 57 (64: »dogmatisch folgerichtig«).

48 Vgl. auch T. Bezzenberger, AcP 196 (1996), 395 (425). 
Einzelfalls einfließen. Bedeutsam ist insbesondere das Gewicht der Diskriminierung. Dafür sind zunächst objektive Umstände heranzuziehen, etwa Dauer, Intensität und Häufigkeit des Verhaltens, die eingesetzten Mittel, die Bekanntgabe gegenüber Dritten, die Bedeutung des Vertragsschlusses für den Betroffenen. Auch die wirtschaftlichen Verhältnisse der Beteiligten sowie das spätere Verhalten des diskriminierenden Marktteilnehmers (insbesondere: Wiedergutmachungsbemühungen) spielen eine Rolle. Daneben können subjektive Momente bedeutsam werden. ${ }^{49}$ Insoweit lässt sich die Sanktion auch nach dem Verschuldensgrad abstufen.

In Betracht kommt zudem, ein präventives Element einfließen zu lassen. Dies liegt im Hinblick darauf nahe, dass die Richtlinien eine »abschreckende« Sanktion verlangen. Zwar sind Strafzwecke dem deutschen Zivilrecht grundsätzlich fremd. Dies bedeutet jedoch nicht, dass über Entschädigungsansprüche nicht auch verhaltenssteuernde Ziele verfolgt werden dürften. Damit ist eine Grundsatzdiskussion ${ }^{50}$ angesprochen, die hier nicht vertieft werden kann. Jedenfalls bei einer Verletzung des allgemeinen Persönlichkeitsrechts - als einem der Diskriminierung sachlich nahestehenden Fall - stellt der BGH ${ }^{51}$ ausdrücklich auch auf die Prävention (Abschreckungswirkung) ab. Dies ist für die Diskriminierungsfälle unter zwei Voraussetzungen sachgerecht: Es muss sich um subjektiv vorwerfbares Verhalten handeln. Zudem dürfen nicht exorbitante Summen nach Art der US-amerikanischen punitive damages angesetzt werden. Von diesen Dimensionen aber ist das deutsche Schadensersatzrecht weit entfernt. ${ }^{52}$

Bemisst man den Entschädigungsanspruch nach den vorstehenden Kriterien, so wird dem Richter ein erheblicher Spielraum gewährt. Man könnte erwägen, diesen Spielraum durch fixe Mindest- oder Höchstbeträge einzuschränken, wie dies in § 613 a III BGB geschehen ist. Indessen können die Umstände von Diskriminierungen sehr unterschiedlich sein, so dass eine derartige Begrenzung nicht sachgerecht erscheint. Zudem zeigt die Rechtsprechungspraxis zur Bemessung von Schmerzensgeld ( 253 II BGB n.F.), dass es durchaus gelingt, Maßstäbe herauszubilden, ${ }^{53}$ wenngleich in den Diskriminierungsfällen das Präventionsinteresse hinzukommt und dieses hier nicht etwa - wie bei Verletzungen des allgemeinen Persönlichkeitsrechts durch Presseorgane - im Wege einer Gewinnabschöpfung verwirklicht werden kann. Eine gewisse Flexibilität bei der Bemessung der Entschädigung stellt aber gerade einen Vorzug dieses Instruments dar, da hierdurch den Besonderheiten des Einzelfalls Rechnung getra-

49 Vgl. Linck, in: Ascheid/Preis/Schmidt, GK-Kündigungsrecht, 2000, § 611 a BGB Rdnr. 103 (zu § 611 a BGB). Abw. insoweit offenbar Baer, ZRP 2001, 500 (502): Es komme nicht auf Einstellungen und Motive, sondern auf »objektiv messbare Folgen« an; vgl. aber auch dies., ZRP 2002, 290 (293).

50 S. etwa Larenz, SchR I, S. 393 ff.; zu § 611 a BGB Herrmann, ZfA 1996, 19 (35 ff.); Linck, in: Ascheid/Preis/Schmidt (Fn. 49), § 611 a BGB Rdnr. 102.

51 BGHZ 128, 1 ff. = NJW 1995, 861; BGH NJW 1996, 984 (985).

$52 \mathrm{Vgl}$. Erman/Ehmann (Fn. 29), Anh § 12 Rdnr. 383; s. auch Wendeling-Schröder, DB 1999, 1012 (1013).

$53 \mathrm{Zu}$ Schmerzensgeldtabellen s. etwa Oetker, in: MünchKomm-BGB, 4. Aufl. 2003, § 253 Rdnr. 37. 
gen werden kann. Auch das Gebot einer wirksamen und abschreckenden Sanktion zwingt nicht zur Einführung starrer Mindestbeträge, ${ }^{54}$ vielmehr haben die Gerichte bei der Konkretisierung der angemessenen Entschädigung die europarechtlich gebotene effektive Sanktionswirkung zu beachten.

\section{Differenzierung zwischen Vertragsschluss und Vertragsbeendigung?}

Bislang wurde der wichtigste Fall einer Diskriminierung betrachtet, nämlich die Verweigerung des Vertragsschlusses. Nun soll noch kurz auf die Ausübung von Gestaltungsrechten eingegangen werden, die zur Beendigung des Vertrages führen. Aus Sicht des Betroffenen erscheint es auf den ersten Blick als gleichgültig, ob ihm schon der Vertragsschluss verwehrt oder ob ein bestehendes Vertragsverhältnis einseitig beendet wird: In beiden Fällen wird eine von ihm gewünschte vertragliche Beziehung abgelehnt. Allerdings besteht auch ein erheblicher Unterschied: Nur im zweiten Fall droht eine Veränderung des status quo (nämlich eines bestehenden Vertragsverhältnisses, z.B. Miete), während im ersten Fall der status quo des Benachteiligten durch die Verweigerung nicht berührt wird. Mit anderen Worten: Nur bei einer einseitigen Beendigung eines Vertrages ist der Bestandsschutz betroffen. Diesem Bestandsschutz kommt aber besonderes Gewicht zu. Das zeigt sich nicht zuletzt an den erhöhten Anforderungen, die für die Kündigung bestimmter Dauerschuldverhältnisse bestehen (Wohnraummiete, Arbeitsvertrag). Es ist daher sachgerecht, die diskriminierende Ausübung von Gestaltungsrechten, die per definitionem in den status quo des Adressaten eingreift, für nichtig zu halten. Einer besonderen Sanktionsanordnung bedarf es insoweit nicht, da das Diskriminierungsverbot ein Verbotsgesetz im Sinne von § 134 BGB ist. ${ }^{55}$

\section{Verhältnis zu allgemeinen Ansprüchen}

Schließlich stellt sich die Frage, in welchem Verhältnis der hier vorgeschlagene Entschädigungsanspruch zu den allgemeinen zivilrechtlichen Ansprüchen steht. Das Diskriminierungsverbot könnte ein Schutzgesetz im Sinne von $§ 823$ II BGB sein. Dies ist dann nicht der Fall, wenn das Gesetz die Rechtsfolgen selbst abschließend regelt. ${ }^{56}$ Gerade eine solche umfassende und abschließende Regelung soll aber nach dem hier unterbreiteten Vorschlag getroffen werden. Sie dient der Rechtsklarheit und damit der Rechtsanwendungssicherheit. Außerdem entfällt, wie europarechtlich vorgegeben, das Verschuldenserfordernis und es wird sichergestellt, dass nicht über die schadensersatzrechtliche Naturalrestitution doch ein Kontrahierungszwang eingeführt wird. Ist die Regelung abschließend, so kommt auch ein konkurrierender Schadensersatzanspruch aus culpa in contrahendo ebenso wenig in Betracht wie ein solcher aus $\S 823$ I BGB

54 In diese Richtung aber Nickel, NJW 2001, 2668 (2671).

55 Vgl. Müller-Glöge, in: MünchKomm-BGB (Fn. 26), § 611 a Rdnr. 53 (zu § 611 a I BGB).

56 Mayer-Maly/Armbrüster, in: MünchKomm-BGB, 4. Aufl. 2001, § 134 Rdnr. 3 (zu § 134 BGB); vgl. auch Müller-Glöge, in: MünchKomm-BGB (Fn. 26), § 611 a Rdnr. 61 m.w.Nachw. (zu § 611 a BGB). 
(Verletzung des allgemeinen Persönlichkeitsrechts) oder aus $§ 826$ BGB. Dasselbe gilt hinsichtlich eines quasi-negatorischen Beseitigungsanspruchs analog § 1004 BGB.

Einer abschließenden Regelung in Gestalt eines Anspruchs auf angemessene Entschädigung in Geld steht schließlich auch nicht die Richtlinienvorgabe ${ }^{57}$ entgegen, wonach die Umsetzung nicht dazu dienen darf, eine Absenkung des »bereits garantierten« Schutzniveaus zu rechtfertigen. Bislang ist ein entsprechendes Schutzniveau nämlich im Bereich diskriminierenden Verhaltens gerade nicht garantiert. ${ }^{58}$ Dies bestätigt zugleich, wie wichtig die ausdrückliche Regelung einer effektive Sanktion ist.

\section{Ergebnisse in Thesen}

1. Bei diskriminierender Ablehnung eines Vertragsschlusses sollte ein Anspruch auf angemessene Entschädigung in Geld und kein Kontrahierungszwang vorgesehen werden.

2. Der Ausgleichsanspruch sollte auch den materiellen Schaden einbeziehen (abschließende Regelung).

3. Bei sonstiger Diskriminierung ist zu unterscheiden: Gestaltungserklärungen sind gem. § 134 BGB unwirksam. In anderen Fällen ist eine angemessene Entschädigung in Geld zu leisten.

57 Art. 6 II der Richtlinie 2000/43/EG; Art. 8 II der Richtlinie 2000/78/EG; Art. 8 e II der Richtlinie 2002/73/EG; Art. 7 II der Richtlinie 2004/113/EG.

58 A.A. die in Fn. Genannten. 